Supplementary Information

\title{
Novel Noncompetitive Type Three Secretion System ATPase Inhibitors Shut Down Shigella Effector Secretion
}

\author{
Heather B. Case ${ }^{1}$, Dominic S. Mattock ${ }^{2}$, Bill R. Miller III ${ }^{2}$ and Nicholas E. Dickenson ${ }^{{ }^{*}}$ \\ ${ }^{1}$ Department of Chemistry and Biochemistry, Utah State University, Logan, UT 84322, USA. \\ ${ }^{2}$ Department of Chemistry, Truman State University, Kirksville, MO 63501, USA. \\ *Corresponding author: Nicholas Dickenson \\ E-mail: nick.dickenson@usu.edu \\ 3 pages (not including cover page) \\ 2 Supplementary Tables
}


Table S1. Properties of T3SS ATPase Inhibitors Evaluated in this Study ${ }^{a}$

\begin{tabular}{|c|c|c|c|c|}
\hline Inhibitor & Structure & $\begin{array}{c}\text { Molecular } \\
\text { Weight } \\
\text { (g/mol) }\end{array}$ & $\log P$ & $\begin{array}{c}\text { Binding } \\
\text { Energy } \\
(\mathrm{kcal} / \mathrm{mol})^{\mathrm{b}}\end{array}$ \\
\hline 8573 & & 462.5 & 2.9 & -11.4 \\
\hline 4967 & & 432.5 & 2.4 & -11.2 \\
\hline 3812 & & 376.4 & 2.2 & -11.0 \\
\hline 6573 & & 473.5 & 1.3 & -11.0 \\
\hline 8771 & & 485.9 & 2.4 & -10.9 \\
\hline 2357 & & 467.9 & 1.9 & -10.7 \\
\hline
\end{tabular}


Table S1 Continued

\begin{tabular}{l|l|l|l|l}
\hline Inhibitor & $\begin{array}{c}\text { Molecular } \\
\text { Weight } \\
(\mathrm{g} / \mathrm{mol})\end{array}$ & $\begin{array}{c}\text { Binding } \\
\text { Energy }\end{array}$ \\
\hline $\mathbf{k n c a l} / \mathrm{mol})^{\mathrm{b}}$
\end{tabular}

aSummary of the properties of the ten predicted Spa47 inhibitors evaluated in this study. Structures, molecular weights, and LogPs were obtained from the manufacturers' websites. 'binding energy refer to the binding energy calculated by QuickVina2 following in silico docking of the inhibitor to Spa47. 
Table S2. T3SS ATPase Inhibitor Binding Sites Display Significant Sequence Conservation ${ }^{\text {a }}$

\begin{tabular}{|c|c|c|c|c|c|c|c|c|c|c|c|c|}
\hline ATPase & Organism & & & & & & esidues & & & & & \\
\hline Spa47 & Shigella & S 250 & Y 254 & L 305 & E 307 & D 313 & P 314 & A 255 & F 279 & L 282 & R 320 & H 326 \\
\hline EscN & E. coli & S 267 & Y 271 & L 321 & E 323 & D 329 & P 330 & A 272 & F 296 & L 299 & R 336 & H 342 \\
\hline Flil & Salmonella & S 273 & Y 277 & L 329 & E 331 & D 337 & P 338 & A 278 & F 302 & L 305 & R 344 & H 350 \\
\hline InvC & Salmonella & S 250 & Y 254 & L 304 & E 306 & D 312 & P 313 & A 255 & F 279 & L 282 & R 319 & H 325 \\
\hline SsaN & Salmonella & S 253 & Y 257 & L 307 & E 309 & E 315 & P 316 & A 258 & F 282 & L 285 & R 322 & H 328 \\
\hline YscN & Yersinia & S 260 & F 264 & L 314 & E 316 & E 322 & P 323 & A 265 & F 289 & L 292 & R 329 & H 335 \\
\hline CdsN & Chlamydia & S 259 & F 263 & L 313 & A 315 & E 321 & P 322 & A 264 & F 288 & L 291 & R 328 & H 334 \\
\hline F1 $\alpha$ & Human & D 313 & Q 317 & E 371 & Q 373 & A 379 & Y 380 & $-b$ & - & - & - & - \\
\hline F1 $\beta$ & Human & - & - & - & - & - & - & Т 312 & A 336 & M 339 & F 376 & T 382 \\
\hline
\end{tabular}

aAlignment of several T3SS ATPase sequences shows strong conservation among residues predicted to surround the interfacial

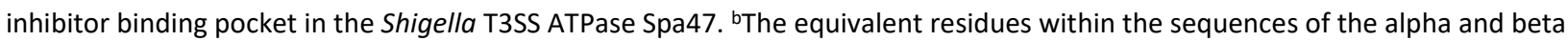
subunits of the non-T3SS hetero-oligomeric F1 ATP synthase are identified and display little-to-no conservation with the T3SS ATPases. 